\title{
CrystEngComm
}

\section{Nanocrystal growth via oriented attachment}

\author{
Cite this: CrystEngComm, 2014, 16, \\ Hengzhong Zhang, ${ }^{* a}$ R. Lee Penn, ${ }^{b}$ Zhang Lin $^{c}$ and Helmut Cölfen ${ }^{d}$ \\ 1407
}

DOI: 10.1039/c4ce90001d

www.rsc.org/crystengcomm

Crystal growth from solution has been classically described as diffusion of ionic or molecular-scale species from the more soluble to the less soluble crystals, as seen in the classical theory of Ostwald ripening. ${ }^{1,2}$ Thus, smaller crystallites serve as nutrients to the larger, growing crystallites. In the last few decades, reports of size-dependent phenomena and materials with novel nano-architectures never before seen have become more frequent. In the late 1990s, then Ph.D. student R. L. Penn in the Banfield group at the University of Wisconsin-Madison studied the hydrothermal coarsening of nanocrystalline titania (anatase). High-resolution transmission electron micrographs of the processed anatase nanocrystals revealed many beautiful necklace-like anatase chains generated by serial attachment across the $\{112\}$ of anatase primary crystallites. ${ }^{3}$ Edge dislocations were also observed in some of the secondary nanocrystals, leading to the discovery of a mechanism by which defects could

\footnotetext{
${ }^{a}$ Department of Earth and Planetary Science, University of California, Berkeley, California 94720, USA. E-mail: heng@eps.berkeley.edu; Fax: +1 510643 9980; Tel: +15106439120

${ }^{b}$ Department of Chemistry, University of Minnesota, 207 Pleasant Street SE, Minneapolis, MN 55455, USA ${ }^{c}$ State Key Laboratory of Structures, Fujian Institute of Research on the Structure of Matter, Chinese Academy of Sciences, Fujian, Fuzhou, 350002, China

${ }^{d}$ Department of Physical Chemistry, University of Konstanz, Universitätsstrasse 10, D-78457 Konstanz, Germany
}

be incorporated into initially defect-free nanoparticles. ${ }^{4}$ This new crystal growth mechanism, distinct from the classical Ostwald ripening, was termed oriented attachment (or oriented aggregation) and provides a route by which unique crystal morphologies and nano-architectures can be produced.

New cutting-edge techniques, such as cryogenic TEM and fluid cell TEM, and availability of more computing power have enabled substantial advances in our fundamental understanding of crystal growth by oriented attachment. Recent work employing fluid cell in situ high-resolution transmission electron microscopy (HRTEM) to directly examine iron oxyhydroxide nanoparticles in water not only directly confirmed the oriented attachment mechanism but also provided fundamental descriptions of the translational and rotational motions of nanoparticles searching for compatible orientations as well as the generation and migration of dislocations during and after attachment. ${ }^{5}$ Oriented attachment, as well as other particle-mediated growth mechanisms, play important roles in the formation of many nano- and/or meso-scale crystals possessing complex morphologies that cannot be explained by classical Ostwald ripening. Experiments tracking. the kinetics of crystal growth in a number of materials have yielded information about rate-limiting steps as well as provided clear evidence that oriented attachment rarely operates as the sole crystal growth mechanism. Computational simulations have enabled improved predictions and understanding through calculations of the energetics and dynamics of oriented attachment. With reports scattered in many different journals, we have assembled this themed issue in CrystEngComm, which focuses on the most recent advances in our understanding of crystal growth by oriented attachment.

In this issue, contributions include reports focusing on experimental and computational approaches. These contributions describe materials including oxides and/or their composites (e.g., $\mathrm{TiO}_{2}, \mathrm{Fe}_{3} \mathrm{O}_{4}, \mathrm{Fe}_{2} \mathrm{O}_{3}, \mathrm{ZnO}$ and $\mathrm{TiO}_{2} / \mathrm{WO}_{3}$ ), oxyhydroxides (e.g., $\mathrm{FeOOH})$, semiconductor compounds (e.g., CdTe), metallic alloys (e.g., $\mathrm{AuCu}$ ), carbonates (e.g., $\mathrm{CaCO}_{3}$ ), and even organic polymers. Advanced techniques, such as cryogenic TEM and synchrotron X-ray diffraction, enable elucidation of oriented attachment in aqueous suspensions or dry powders at elevated temperatures. The kinetics of oriented attachment at different conditions, and the controlling factors, have been reviewed and analyzed. Molecular dynamics simulations have provided insights into the details of oriented attachment at the atomic level. The thermodynamic and physical driving forces for oriented attachment have been revisited and revised based on new atomistic calculations. Thus, this issue provides a holistic description of the state-of-the-science research 
focused on crystal growth by oriented attachment.

With the collaborating efforts of the guest and CrystEngComm editors and the contributing authors, this themed issue is now presented to the crystal growth community. We hope this issue will inspire new research in many areas, providing new knowledge and enhancing our ability to make use of it. Myriad opportunities exist in areas such as advanced functional nanomaterials prepared by exploiting oriented attachment through both computational design and experimental exploration; sampling, surveying, and identifying naturally occurring (or environmental) nanoparticles formed by oriented attachment; investigation of the atomic details of oriented attachment by developing and applying new characterization/ analytical tools; and fabrication of composite nano/bio-materials by exploiting oriented attachment for a wide range of applications (such as for bio-medicals and electronics).

\section{References}

1 W. Ostwald, Lehrbuch der Allgemeinen Chemie, vol. 2, Part 1., Engelmann, Leipzig, Germany, 1896.

2 W. Ostwald, Z. Phys. Chem., 1897, 22, 289.

3 R. L. Penn and J. F. Banfield, Geochim. Cosmochim. Acta, 1999, 63, 1549.

4 R. L. Penn and J. F. Banfield, Science, 1998, 281, 969.

5 D. Li, M. H. Nielsen, J. R. I. Lee, C. Frandsen, J. F. Banfield and J. J. De Yoreo, Science, 2012, 336, 1014. 